\title{
An educational environment based on digital image processing to support the learning process of biomaterials degradation in stem cells
}

\author{
Y. Robles-Bykbaev*†t, S. Naya†, J. Tarrío-Saavedra*, S. Díaz-Prado*, C. Sanjurjo*, F. \\ Blanco* \\ * Dpto. de Matemáticas, Universidade da Coruña, Spain \\ † INIBIC, SERGAS, Complejo Hosp. Univ.,Universidade da Coruña, Spain \\ Email: zrobles@ups.edu.ec, \{salva,javier.tarrio\}@udc.es, \{s.diaz1,biocsr00\}@udc.es,ffblagar@sergas.es
}

\section{J. Cornejo-Reyes, L. Garzón, D. Calle-López, V. Robles-Bykbaev}

+ GI-IATa, GiMat, Cáatedra UNESCO Tecnologías de apoyo para la Inclusión Educativa, Universidad Politécnica Salesiana, Cuenca, Ecuador

Email:pjaviercornejo@gmail.com, \{lgarzon,dcallel,vrobles\}@ups.edu.ec

\begin{abstract}
:
The Poly(DL-lactide-co-glycolide) copolymers (PDLGA) have designed and performed as biomaterials, taking into account their biodegradability and biocompatibility properties. These materials have a wide range of application in medicine such as orthopedic implants, general surgical implants (suture materials), osteosynthesis, bone cement, among many others. For these reasons, in this paper, we present an intelligent educational environment that can be used for both, researchers and students interested in the analysis of the biomaterial behavior under certain conditions. Our platform includes a Learning Objects (LOs) for MOODLE, and in the same way, implements several digital image processing techniques as well as a decision support module based on a random forest algorithm and statistical modeling. With the aim of determining the real feasibility of this proposal, we have presented the system to 34 Ecuadorian engineering students. After testing the platform, the students answered a survey aimed at determining their perception of the system. The results provide several guidelines to continue with the developing of the platform.
\end{abstract}

Keywords

Biomaterials degradation; Computer Vision; MOODLE; Decision support systems 


\section{INTRODUCTION}

Several authors and professionals emphasize how e-learning is considered clear evidence of academic effectiveness for several key reasons [1]. First, electronic learning technologies. Second, the role it plays in education. Third, the evaluation strategies. All of these reasons and many others, express the effectiveness of online learning platforms, complementing the learning objectives achieved within the traditional educational schemes.

Referring to the first reason, the learning platforms developed through these technologies allow the student to have a better control over the content, the optimization of their learning time and their adaptation to the different academic objectives. The second reason refers to the easy access to academic resources such as digital libraries and e-learning materials that support the development of research and finally, it is not considered that the online learning platforms replace the teacher (traditional system) but this is a complement to the learning assessment strategies [2].

The innovations that are given trough online educational environments show a better way to reach students so that, based on their different learning styles, they can take advantage of its contents to contribute to improving the quality of their knowledge, this represents a great improvement in the quality of education and in the improvement of the overall performance of students.

There is evidence for the effectiveness and acceptance of e-learning within the medical education community, especially when combined with traditional teacher-led activities in a blended-learning educational experience [2]. The use of the educational environment facilitates both students and researchers a better understanding of the machine learning techniques presented as well as the correct statistical modeling for these systems, thus gaining knowledge about concepts that can be easily extrapolated and applied on other types of analysis.

In the same line, it is important to mention that nowadays the biomaterials are widely used in a wide range of applications for medical purposes such as the following: joint replacements, bones plates, artificial ligaments and tendons, cochlear implants, dental implants for tooth fixation, among many other uses.

For these reasons, in this paper, we present a novel approach that focuses on providing an educational environment where both students and experts can study the process of degradation of biomaterials through image analysis, artificial intelligence, and statistical modeling. In this stage, we present a specific case focused on the degradation analysis of collagen type-I in stem cells and how the engineering students perceive the functionalities provided by the platform.

The rest of the paper is organized as follows. In Section 2 we present a brief overview of the importance of using educational platforms as well as the problem-based learning. The general architecture of the proposed system is described in Section 3. The pilot experiment carried out with 34 Ecuadorian engineering students as well as its preliminary results are presented in Section 4. Finally, Section 5 presents the conclusions and some ideas for future work. 


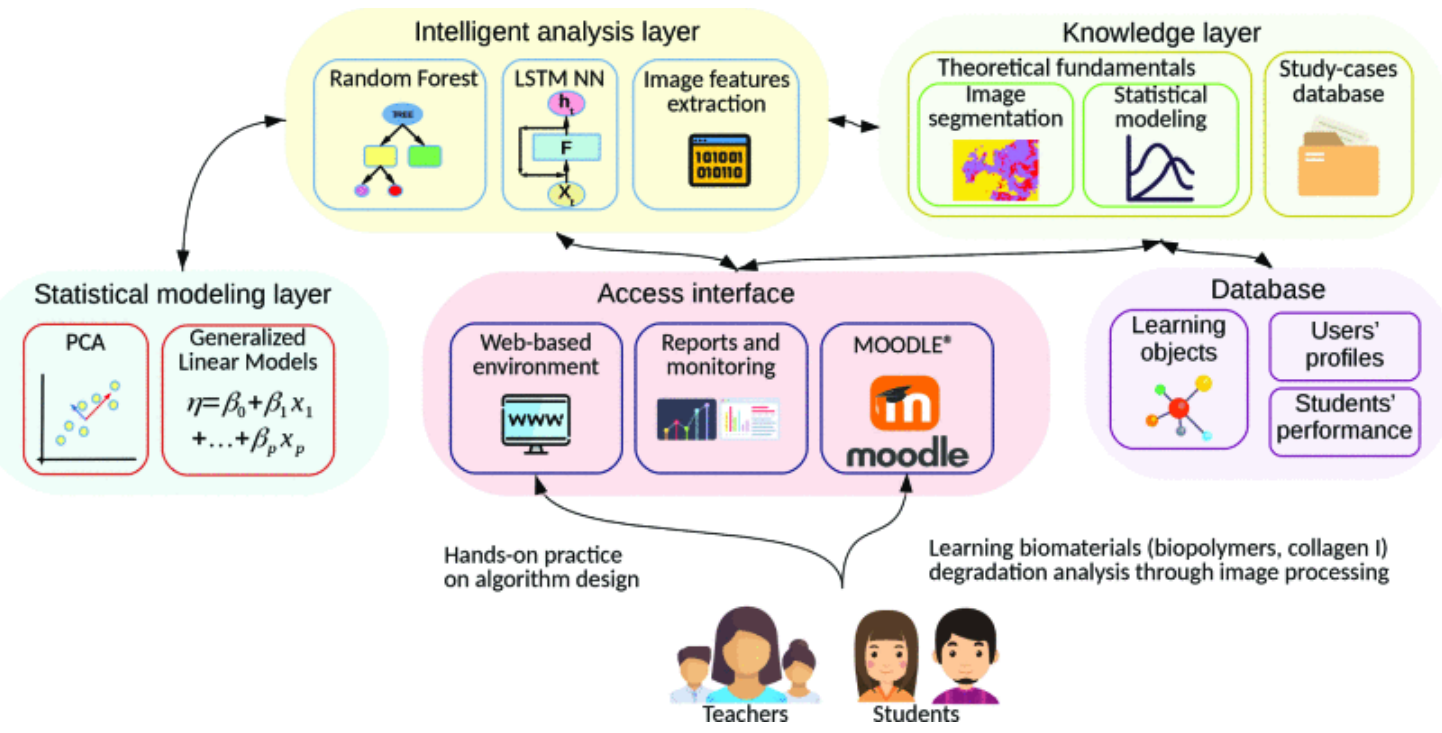

Fig. 1. The main modules and components that make up the proposed educational environment.

\section{RELATED WORK}

The teaching-learning process has a heugh improvement in this new era through the use of the Information and Communication Technologies (ICTs) for example the educational platforms. This "telematic tools can be considered as an indicator of the development of society" [3]. Educational platform as a telematic tool allow the diversification of knowledge, the flexibility of the teaching-learning process, stimulate creativity and promote collaboration [3].

Educational platforms are considered part of several Learning Management Systems (LMS). "Electronic learning is a technology learning that plays an important role in modern education and training" [4]. In that way is considered that the educational platform as digital tool can contribute to the educational inclusion processes because "the fact that content is available at any place, it means that the education is accessible everywhere with the use of this technology" [4].

Open-source platforms for bachelors educational can have a heugh contribution over the educational area "because it can be freely inspected, modified, and redistributed; in particular, open software's as platforms like ImageJ has had a huge impact on the life sciences, and continues to do so" [5].

There is another approach to learning object based on problem-based learning [6], in this approach learning units are created based on simulations of patient cases. The proposed cases are the result of what has been experienced and learned in real cases, in this way the teacher can specify both the areas of study and the characteristics of the problem, thus achieving that students have relevant challenges to improve their self-learning ability, resulting in the development of essential skills to make correct diagnoses. 


\section{GENERAL SYSTEM ARCHITECTURE}

Our main propose is to provide detailed protocols step by step to learn the images segmentation of mesenchymal stem cells growth in biomaterials as collagen type-I through the use of representative images of proper segmentation and the quantitative data obtained through Random Forest segmentation technique. Our platforms, as proposes [5], is an accessible learning tool "for everyday use in life science and to develop the technology further". Figure 1 depicts the different layers and components that make up our system. Bellow, we describe the most relevant characteristics of each layer:

- The users (teachers and students) can interact with the platform through the access interface layer. This layer provides the following elements: a) a web-based environment where the students can practice and experiment with the design of algorithms to perform the image processing, b) a MOODLE instance that includes educational contents and evaluations, and c) a module for generating reports of students progress.

- The database layer stores the information related to the users' profiles as well as the results achieved by students during the practical activities and contents' learning.

- In the statistical modeling layer, we have implemented several algorithms to support the analysis of several features related to biomaterials' degradation. For example, the Principal Component Analysis (PCA) and the Generalized Linear Models (GLM) [7] allow studying the relations that exist among different variables as area, eccentricity, ration aspect, etc. of the biomaterials artifacts.

For example, currently the system can perform several GLM such as the following: collagen degradation regards time + cell culture group (time effects, cell group in the degradation of type I collagen).

With this model, where $\eta=\mu_{x}=\beta_{o}+\beta_{1} \cdot x_{1}($ T ime $)+\beta_{2} \cdot x_{2}$ (group), $\mu_{x}$ follows a normal distribution. The variance proportion explained in the model (Residual deviance) was apparently small $(4.0879 \mathrm{e}-05)$ and the Akaike Information Criterion (AIC) [8] was 1454.2.

To test $H 0: \beta_{0}=0$, we use $z=2.049(p$ value $=2.70 e-08)$. Consequently, the cell culture - group, regards to time, seems to have a meaningful impact on the probability of the type I collagen degradation after time goes (i.e. once the model includes that variable "glm. without. network"). Namely, that model has the best p-value for time and the group, and the smallest values for AIC and Residual deviance compared to the other proposed models

- The intelligent analysis layer contains several functionalities to support the process related to the image's segmentation and features extraction. Likewise, this layer implements the following algorithms: Random Forest [9] for image segmentation, Long Short-Term Memory (LSTM) Neural Networks [10] and functionalities to extract image's features (histograms, areas, etc.).

- In the knowledge layer, are defined the theoretical fundamentals to perform the image segmentation (regions of interest) and the statistical modeling of biomaterial artifacts (collagen degradation, etc.). Similarly, this layer contains a study-cases database related to the guides provided by teachers and experiments carried out by students. 


\section{EXPERIMENT AND RESULTS}

As the first step, the educational platform was developed and implemented in eXeLearning (http://exelearning.net). We have uploaded contents and the respective instructions to learn how is the segmentation biological images process step by step. The content was divided in five sections in order to offer a user-friendly platform for a better student learning process. For example, Figure 2 depicts two screen captures of the platform. The upper image shows an educational content that addresses the cell differentiation based on structure tensors and entropy. On the other hand, the bottom image describes an evaluation example of Random Forest as a technique for image segmentation.

Additional to the developed platform, we have applied a survey to students from 5th to 10th levels of study. The participants were students from 20 to 26 years old from informatics, telematics, electronic and mechanical engineering careers. The survey was previously tested through Cronbach's alpha (an index of reliability) [11]. Cronbach's alpha index was about 0.84 . Which means that the reliability index of the construct. In this stage we tested 34 engineering students previously described, from the "Universidad Politécnica Salesiana" in Cuenca-Ecuador, to know their perceptions about this educational platform to learn the images segmentation of mesenchymal stem cells growth in biomaterials as collagen type-I through the use of representative images of proper segmentation and the quantitative data obtained through Random Forest segmentation technique.

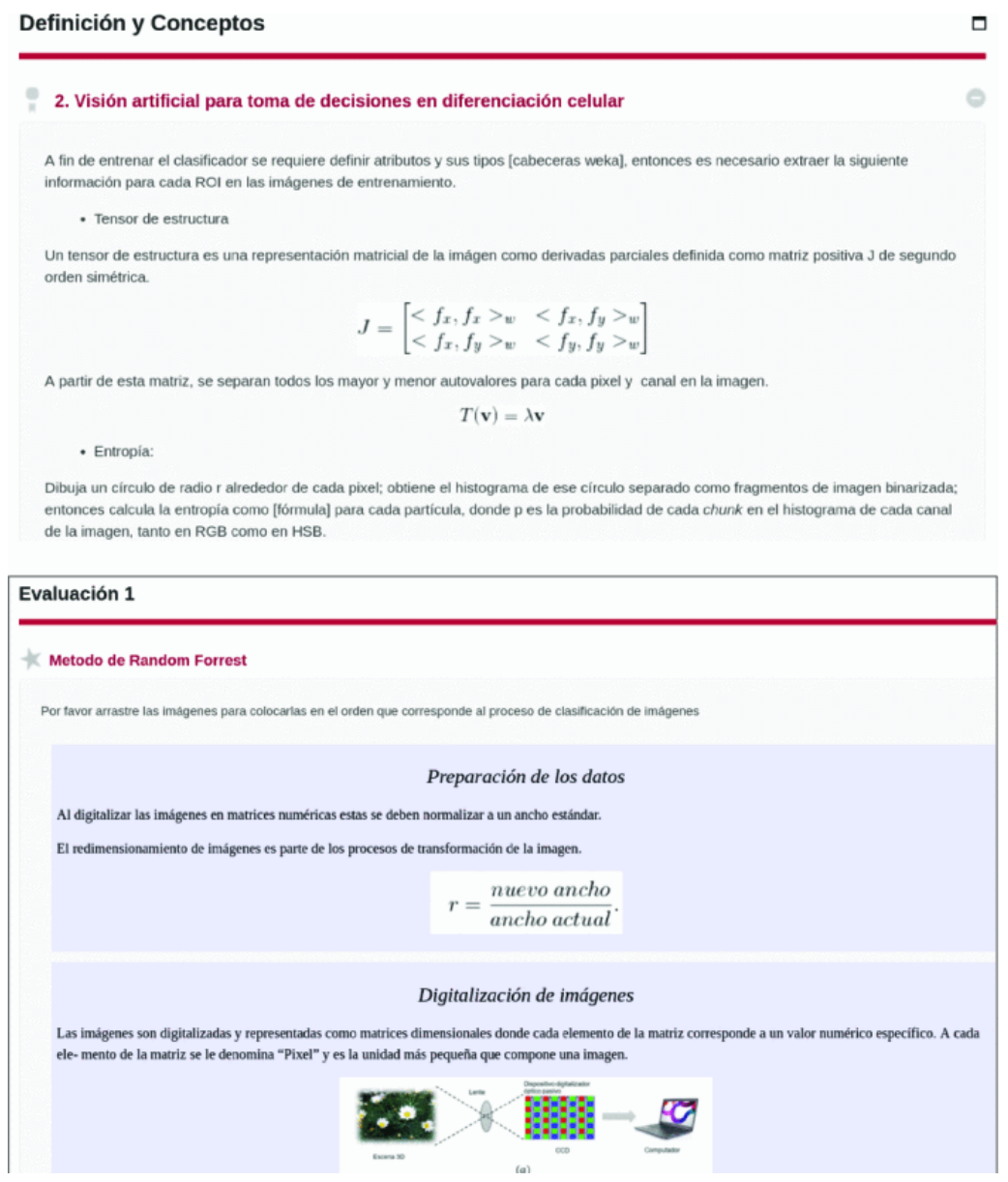

Fig. 2. Two screen captures of an educational content related to decision-making support in images segmentation (upper image) and an example of an evaluation of segmentation methods (bottom image). 


\section{A. Results}

In relation to the described students sample, we observed that the female engineering students from 3 th and 4th study semester, does not use frequently images segmentation ("never" and "hardly never"). From other side, male surveyed students from 4th and 5th semester study form informatics and electronic engineering also does not use frequently images segmentation ("never" and "hardly never") unlike the other professions (mechanical technician and telematics engineering) who describe that they has use very frequently the images segmentation ("always", "very frequently", "frequently"), with means that male part from sample surveyed may be has more knowledge about this technological tool to Learning Management Systems.

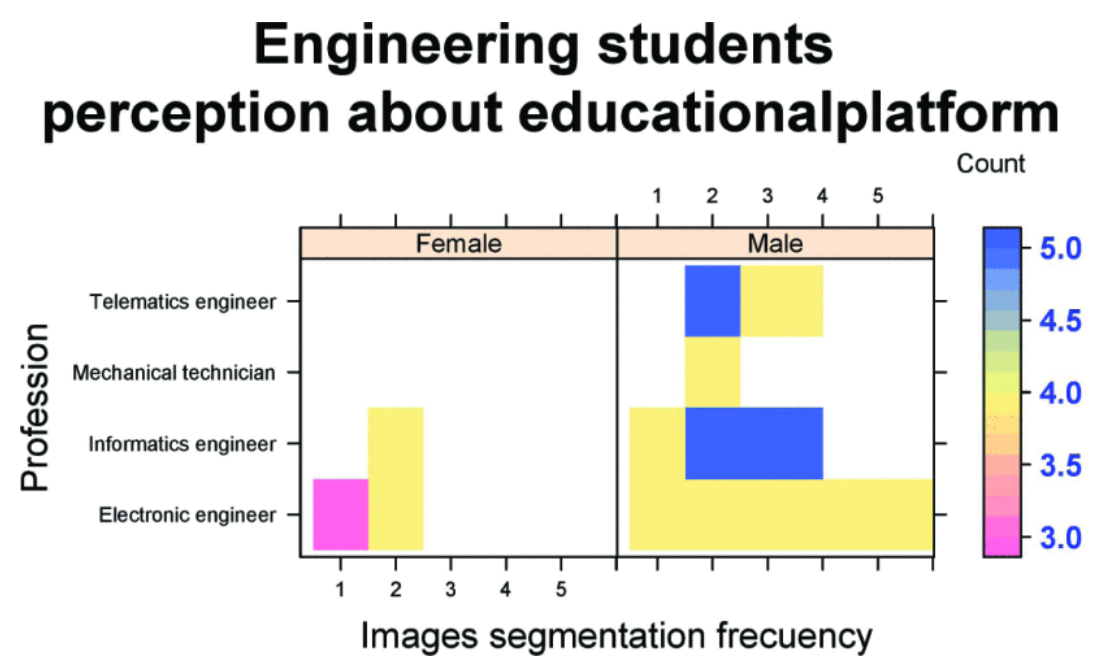

Fig. 4. Engineering students male and female perception about the easy-to use of images segmentation. 


\section{CONCLUSION}

In this paper, we have presented a brief overview of a new educational platform to support the learning biomaterials degradation analysis through image processing and statistical modeling. The primary objective of this research was to determine the real feasibility of using this tool in real cases where engineering students must analyze images.

As for the results of the surveys, we can conclude that profession has a significant influence on the images segmentation frequency and easy-to use. As is traditional in our Ecuadorian educational context, we observed through this study that we have a poor statistical presence of female students in this engineering careers.

Also, we can say that this educational platform is very important to improve the educational process of engineering students related to the area of Learning Management Systems as TICs tools in the inclusive educational process of early bachelor carriers.

As lines of future work we propose the following ones:

- To develop a module to automatically inject new algorithms designed by students to carry out the segmentation image analysis and the statistical modeling.

- To develop a decision-making support module that can provide guidelines to apply the different operations required to carry out the images segmentation as well as the region of interest extraction. 


\section{ACKNOWLEDGMENT}

This project was partially funded by the "Ministerio de Educación y Ciencia MTM2014-59543-P", also by the "Sec-retara Nacional de Educación Superior, Ciencia, Tecnología e Innovación del Ecuador (SENESCYT)". The authors from the Universidad Politécnica Salesiana have been supported by the "Sistemas Inteligentes de Soporte a la Educación (v5)" research project, the Cátedra UNESCO "Tecnologías de apoyo para la Inclusión Educativa" initiative, and the Research Group on Artificial Intelligence and Assistive Technologies (GI-IATa).

\section{REFERENCES}

[1] R. C. Clark, R. E. Mayer, E-learning and the science of instruction: Proven guidelines for consumers and designers of multimedia learning, John Wiley \& Sons, 2016.

[2] J. G. Ruiz, M. J. Mintzer, R. M. Leipzig, "The impact of e-learning in medical education", Academic medicine, vol. 81, no. 3, pp. 207-212, 2006.

[3] O. Benítez Menéndez, L. Arias Verdecia, Curso virtual de historia de Cuba sobre la Plataforma Educativa Cubana ZERA, 2018.

[4] R. J. M. Ventayen, K. L. A. Estira, M. J. De Guzman, C. M. Cabaluna, N. N. Espinosa, "Usability evaluation of google classroom: Basis for the adaptation of gsuite e-learning platform", Asia Pacific Journal of Education Arts and Sciences, vol. 5, no. 1, 2018.

[5] J. Schindelin, C. T. Rueden, M. C. Hiner, K. W. Eliceiri, "The imagej ecosystem: An open platform for biomedical image analysis", Molecular reproduction and development, vol. 82, no. 7-8, pp. 518-529, 2015.

[6] H. S. Barrows, R. M. Tamblyn et al., Problem-based learning: An approach to medical education, Springer Publishing Company, 1980.

[7] J. M. Chambers, "Linear models" in Statistical models in S, Routledge, pp. 95-144, 2017.

[8] S. N. Wood, Generalized additive models: an introduction with R, Chapman and Hall/CRC, 2006.

[9] D. Mahapatra, "Analyzing training information from random forests for improved image segmentation", IEEE Transactions on Image Processing, vol. 23, no. 4, pp. 1504-1512, 2014.

[10] K. Amarasinghe, D. L. Marino, M. Manic, "Deep neural networks for energy load forecasting" in Industrial Electronics (ISIE) 2017 IEEE 26th International Symposium on, IEEE, pp. 14831488, 2017.

[11] J. R. A. Santos, "Cronbachs alpha: A tool for assessing the reliability of scales", Journal of extension, vol. 37, no. 2, pp. 1-5, 1999. 